\title{
Gas-Particle Flow in a De Laval Nozzle with Curved Convergent Configuration
}

\author{
Yichuan PENG and Ting HAN \\ Department of Thermal Energy Engineering, Northeastern University, Shenyang, Liaoning, 110006 P.R.C.
}

(Received on June 12, 1995; accepted in final form on September 25, 1995)

\begin{abstract}
An analytical procedure of gas-particle mixture flow in the De Laval nozzle is described for the case where the curved convergent part of nozzle has been prescribed. The effect of particle volume fraction, particle clouds, and nozzle configuration on the gas-particle flow is analytically considered. According to the Mach number checkout method, the critical cross-sectional area of the nozzle and the behavior of the supersonic gas-particle flow were determined and calculated respectively.
\end{abstract}

KEY WORDS: gas-particle flow; De Laval nozzle; curved convergent configuration; particle clouds; Mach number checkout method; critical cross-sectional area.

\section{Introduction}

The flow behavior of gas-particles in the nozzle has paramount importance because it is utilized in a wide range of various industrial applications such as aerospace, metallurgical and chemical industries. The analysis of nozzle flow of gas-particle mixture for achieving the optimum nozzle performance is of importance. Therefore, the problem of gas-particle flow through a nozzle has been considered by many authors. ${ }^{1-9)}$ However, many details remain unknown.

Neilson, ${ }^{1)}$ Zucrow and Hoffman ${ }^{2)}$ have described the system of equations governing the steady, quasi-onedimensional flow of gas-particle mixture. In this paper, the above system of equations has been rearranged so that the system gives a reasonable application to the De Laval nozzle. It is noted that all the theoretical calculations of Refs. ${ }^{1-5)}$ have not considered the effect of the volume occupied by the particles on the gas pressure. In fact, when the ratio of mass flow rate of particles to the total mass flow rate is large, the influence of the particle volume fraction must be considered. Therefore, the system of equations presented here considers the influence of particle volume fraction on the behavior of gas-particle mixture.

In some studies, ${ }^{2-3)}$ the pressure distribution was considered as the known parameter, while the nozzle geometry was determined in accordance with the given pressure distribution. In other studies, ${ }^{6-9)}$ the nozzle geometry was previously given, while the critical cross-section area of the nozzle was determined by the pressure checkout method. In this study, the nozzle geometry was still previously given, but a new method - the Mach number checkout method- is used for determining the critical cross-section. As compared with the specified area method and the specified pressure method, this new method has higher accuracy of calculation and requires less computational time.

In the present paper, a mathematical model of gas-particle mixture through a De Laval nozzle with curved convergent configuration has been developed. At the same time, the effect of particle clouds and nozzle configuration on the gas-particle flow is considered. Especially, the effect of different parameters for the cured convergent part of the De Laval nozzle on the behavior of supersonic flow of gas-particle mixtures has been discussed in detail. It is noted that the present calculated results have been partly verified by the experiment in the Ref. ${ }^{7)}$

\section{Mathematical Model}

\subsection{Basic Assumptions}

The following assumptions are introduced:

1. The flow is considered to be steady and quasi-onedimensional; 2 . The gas-particle flow is considered to be independent of the external agencies; 3. The gas is considered to be as perfect gas; 4 . The particles are considered to be spherical in shape and incompressible; 5 . The density and specific heat of particles are considered to be constant; 6 . The particle clouds exerts a drag force on the gas, and they transfer the heat to the gas.

\subsection{Configuration of Nozzle}

In the present model, the configuration of De Laval nozzle is shown in Fig. 1. The convergent part of nozzle is made by the rotation of a circle arc which radius of curvature is $R$. The divergent part of nozzle is conic. The angle between arc tangent at the inlet of nozzle and axis of nozzle is $\theta_{1}$ (i.e. initial tangent angle), the cone angle of divergent part is $\theta_{2}$. According to geometry of nozzle, the equations of cross-sectional area for convergent and 


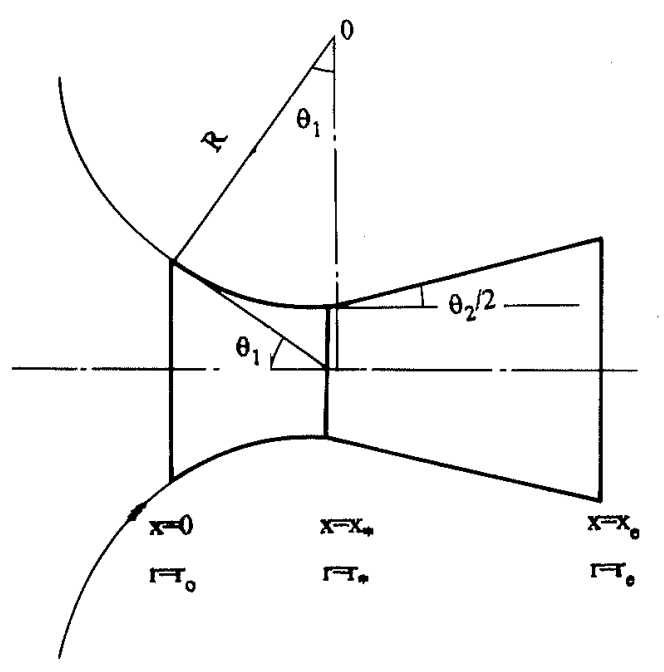

Fig. 1. Sketch of De Laval nozzle.

divergent part of De Laval nozzle are derived as follows:

$$
\begin{gathered}
A=\pi\left[r_{0}+R \cos \theta_{1}-\sqrt{R^{2}-\left(R \sin \theta_{1}-x\right)^{2}}\right]^{2} \\
0 \leq x \leq x_{*} \\
A=\pi\left[r_{*}+\left(x-x_{*}\right) \operatorname{tg}\left(\theta_{2} / 2\right)\right]^{2} \\
x_{*} \leq x \leq x_{\mathrm{e}}
\end{gathered}
$$

It is noted that the cross-sectional area $A$ of arc convergent part is dependent on the radius of curvature $R$, angle $\theta_{1}$ and radius $r_{0}$. The influence of nozzle configurations on the behavior of gas-particle are considered.

\subsection{Governing Equations}

The governing equations describing the gas-particle flows behavior can be derived in accordance with laws of the fluid mechanics as follows.

The mass conservation laws of gas and particles, respectively, are given by

$$
\begin{aligned}
& \alpha \rho_{\mathrm{g}} U_{\mathrm{g}} A=(1-\lambda) W \\
& (1-\alpha) \rho_{\mathrm{p}} U_{\mathrm{p}} A=\lambda W
\end{aligned}
$$

The momentum conservation laws of gas and particles, respectively, are given by

$$
\begin{gathered}
\alpha \rho_{\mathrm{g}} U_{\mathrm{g}} \frac{d U_{\mathrm{g}}}{d x}+(1-\alpha) \rho_{\mathrm{p}} U_{\mathrm{p}} \frac{d U_{\mathrm{p}}}{d x}=-\frac{d p}{d x} \\
\rho_{\mathrm{p}} U_{\mathrm{p}} \frac{d U_{\mathrm{p}}}{d x}=\frac{3}{4} \frac{C_{\mathrm{d}}}{d_{\mathrm{p}}} \rho_{\mathrm{g}}\left(U_{\mathrm{g}}-U_{\mathrm{p}}\right)^{2} \ldots \ldots
\end{gathered}
$$

In which the drag coefficient $C_{\mathrm{d}}$ is expressed as ${ }^{10)}$

$$
\begin{gathered}
C_{\mathrm{d}}=\frac{24}{R e}\left(1+0.125 R e^{0.72}\right) \psi \quad R e<1000 \\
C_{\mathrm{d}}=0.4454 \quad 1000<\operatorname{Re}<3.5 \times 10^{5}
\end{gathered}
$$

It is noted that the effect of the particles cloud on the drag coefficient of an individual particle must also depend on the particle volume fraction $(1-\alpha)$ which is occupied by particle. A lengthy statistical analysis by Tam ${ }^{11)}$ yields a correction factor for drag coefficient as follows:

$$
\psi=\frac{7-3 \alpha+3\left[5-2 \alpha-3 \alpha^{2}\right]^{1 / 2}}{(3 \alpha-1)^{2}}
$$

It has also been suggested, as discussed by Zuber, ${ }^{12)}$ that the particle Reynolds number should be based on a gas viscosity for the particle volume, an effective viscosity is given by

$$
\mu_{\mathrm{e}}=(3.5-2.5 \alpha) \mu_{\mathrm{g}}
$$

Thus, Reynolds number is given by

$$
R e=\frac{\rho\left(U_{\mathrm{g}}-U_{\mathrm{p}}\right) d_{\mathrm{p}}}{\mu_{\mathrm{e}}}
$$

The energy conservation laws of gas and particles, respectively, are given by

$$
\begin{aligned}
& \alpha \rho_{\mathrm{g}} U_{\mathrm{g}}\left(U_{\mathrm{g}} \frac{d U_{\mathrm{g}}}{d x}+C_{\mathrm{pg}} \frac{d T_{\mathrm{g}}}{d x}\right)+(1-\alpha) \rho_{\mathrm{p}} U_{\mathrm{p}} \\
& \left(U_{\mathrm{p}} \frac{d U_{\mathrm{p}}}{d x}+C_{\mathrm{s}} \frac{d T_{\mathrm{p}}}{s x}\right)=0 \\
& \rho_{\mathrm{p}} U_{\mathrm{p}} C_{\mathrm{s}} \frac{d T_{\mathrm{p}}}{d x}=-N u \frac{6 K_{\mathrm{g}}}{d_{\mathrm{p}}^{2}}\left(T_{\mathrm{p}}-T_{\mathrm{g}}\right)
\end{aligned}
$$

In which Nusselt number $N u$ is expressed as

$$
N u=2.0+0.6 \operatorname{Re}^{1 / 2} \operatorname{Pr}^{1 / 3}
$$

The equations of state for the gas and particles, respectively, are given by

$$
\begin{aligned}
& \rho_{\mathrm{g}}=\frac{P}{R_{\mathrm{g}} T_{\mathrm{g}}} \\
& \rho_{\mathrm{p}}=\text { const. }
\end{aligned}
$$

Mach number is expressed as

$$
M=\frac{U_{\mathrm{g}}}{\left(\gamma R_{\mathrm{g}} T_{\mathrm{g}}\right)^{1 / 2}}
$$

The nine aforementioned equations may be rearranged algebraically as follows, to obtain expressions for the numberical calculation of all flow properties ${ }^{7)}$ :

$$
\begin{array}{r}
\frac{d U_{\mathrm{p}}}{d x}=\frac{3}{4} \frac{C_{\mathrm{d}}}{d_{\mathrm{p}}} \frac{\rho_{\mathrm{g}}}{\rho_{\mathrm{p}}} \frac{\left(U_{\mathrm{g}}-U_{\mathrm{p}}\right)^{2}}{U_{\mathrm{p}}} \ldots \ldots \ldots \ldots \ldots(10) \\
\frac{d T_{\mathrm{p}}}{d x}=-\frac{6 K_{\mathrm{g}}}{\rho_{\mathrm{p}} C_{\mathrm{s}} d_{\mathrm{p}}^{2} U_{\mathrm{p}}} N u\left(T_{\mathrm{p}}-T_{\mathrm{g}}\right) \ldots \ldots \ldots \ldots . . . \ldots \ldots \ldots \\
\frac{T_{\mathrm{g}} R_{\mathrm{g}}}{\alpha A} \frac{d A}{d x}+\frac{\lambda}{1-\lambda} \frac{C_{\mathrm{s}}}{C_{\mathrm{pg}}} R_{\mathrm{g}} \frac{d T_{\mathrm{p}}}{d x} \\
\frac{d U_{\mathrm{g}}}{d x}=\frac{-\left[\frac{\lambda}{1-\lambda}\left(\alpha U_{\mathrm{g}}-\frac{U_{\mathrm{p}} R_{\mathrm{g}}}{C_{\mathrm{pg}}}\right)-\frac{1-\alpha}{\alpha} \frac{T_{\mathrm{g}} R_{\mathrm{g}}}{U_{\mathrm{p}}}\right] \frac{d U_{\mathrm{p}}}{d x}}{U_{\mathrm{g}}\left(\alpha-\frac{R_{\mathrm{g}}}{C_{\mathrm{pg}}}-\frac{R_{\mathrm{g}} T_{\mathrm{g}}}{U_{\mathrm{g}}^{2}}\right)} \\
\frac{d T_{\mathrm{g}}}{d x}=\frac{T_{\mathrm{g}}}{\alpha A} \frac{d A}{d x}-\left(\frac{\lambda}{1-\lambda} \frac{\alpha U_{\mathrm{g}}}{R_{\mathrm{g}}}-\frac{1-\alpha}{\alpha} \frac{T_{\mathrm{g}}}{U_{\mathrm{p}}}\right) \frac{d U_{\mathrm{p}}}{d x}
\end{array}
$$




$$
\begin{aligned}
& -U_{\mathrm{g}}\left(\frac{\alpha}{R_{\mathrm{g}}}-\frac{T_{\mathrm{g}}}{U_{\mathrm{g}}^{2}}\right) \frac{d U_{\mathrm{g}}}{d x} \\
& \frac{d \alpha}{d x}=\frac{1-\alpha}{U_{\mathrm{p}}} \frac{d U_{\mathrm{p}}}{d x}+\frac{1-\alpha}{A} \frac{d A}{d x} \\
& \frac{d p}{d x}=p\left(\frac{1}{T_{\mathrm{g}}} \frac{d T_{\mathrm{g}}}{d x}-\frac{1}{U_{\mathrm{g}}} \frac{d U_{\mathrm{g}}}{d x}-\frac{1}{A} \frac{d A}{d x}-\frac{1}{\alpha} \frac{d \alpha}{d x}\right) \\
& \frac{d \rho_{\mathrm{g}}}{d x}=\rho_{\mathrm{g}}\left(\frac{1}{p} \frac{d p}{d x}-\frac{1}{T_{\mathrm{g}}} \frac{d T_{\mathrm{g}}}{d x}\right) \\
& \frac{d M}{d x}=M\left(\frac{1}{U_{\mathrm{g}}} \frac{d U_{\mathrm{g}}}{d x}-\frac{1}{2 T_{\mathrm{g}}} \frac{d T_{\mathrm{g}}}{d x}\right)
\end{aligned}
$$

The present calculation is based on the geometry of nozzle as sketched in Fig. 1. With the help of the equations of cross-sectional area as discussed earlier, the final form of the equations can be obtained by

$$
\begin{aligned}
& \frac{d A}{d x}=-2 \pi\left(R \sin \theta_{1}-x\right)\left\{\frac{r_{0}+R \cos \theta_{1}}{\left[R^{2}-\left(R \sin \theta_{1}-x\right)^{2}\right]^{1 / 2}}-1\right\} \\
& 0 \leq x \leq x_{*} \\
& \frac{d A}{d x}=2 \pi \operatorname{tg} \frac{\theta_{2}}{2}\left[r_{*}+\left(x-x_{*}\right) \operatorname{tg} \frac{\theta_{2}}{2}\right] \\
& x_{*} \leq x \leq x_{\mathrm{e}}
\end{aligned}
$$

It can been seen that Eqs. (10)-(18) constitute a closed set of first-order ordinary differential equations for nine dependent variables $U_{\mathrm{p}}, T_{\mathrm{p}}, U_{\mathrm{g}}, T_{\mathrm{g}}, \alpha, p, \rho_{\mathrm{g}}, M$ and $A$. If the initial conditions and transport properties of gas-particle flow are given, then solutions of the set of these equations can be obtained numerically.

As Ref. ${ }^{6)}$ pointed out, when the cone angle of divergent part $\theta_{2}$ is larger than critical cone angle $\theta_{\mathrm{c}}$, it is possible to get supersonic flow in the divergent part of De Laval nozzle. The critical cone angle $\theta_{\mathbf{c}}$ can be calculated as follows:

$$
\begin{aligned}
\theta_{\mathrm{c}}= & 2 \operatorname{arctg}\left[\frac { \alpha _ { * } D _ { * } } { 4 } \left\{\frac{3}{4} C_{\mathrm{d} *} \frac{\rho_{\mathrm{g} *}}{\rho_{\mathrm{p}}} \frac{\left(1-K_{*}\right)^{2}}{K_{*}} \frac{1}{d_{\mathrm{p}}} \frac{\lambda}{1-\lambda}\right.\right. \\
& \times\left[\alpha_{*} \gamma M_{*}^{2}-(\gamma-1) M_{*}^{2} K_{*}-\frac{1-\alpha_{*}}{\alpha_{*}} \frac{1-\lambda}{\lambda} \frac{1}{K_{*}}\right] \\
& \left.\left.+6 \frac{N u_{*}}{\operatorname{Pr}} \frac{\lambda}{1-\lambda}\left(\frac{T_{\mathrm{p} *}}{T_{\mathrm{g} *}}-1\right) \frac{\mu_{\mathrm{g} *}}{\rho_{\mathrm{p}} U_{\mathrm{p} *} d_{\mathrm{p}}^{2}}\right\}\right]
\end{aligned}
$$

\subsection{Mach Number Checkout Method}

It has been suggested by many authors, ${ }^{2-3}$ ) when the supersonic flow is considered for the previously prescribed nozzle geometry, the specified area method can be employed for upstream as well as downstream of the transonic region, and the specified pressure method can be employed in the transonic region. In other works, ${ }^{6-8)}$ the pressure checkout method is used in the numerical analysis of the gas-particle flow in the De Laval nozzle.

In the present model, Mach number checkout method is used to determine the critical cross-section. When the supersonic flow exists in the divergent part of De Laval nozzle, the Mach number should be increased along with nozzle axis. Therefore, the critical cross-section could be determined by the Mach number checkout method only using the condition of $M_{2}<M_{1}$. Here, $M_{1}$ and $M_{2}$ are the Mach number at the previous step and the following step of the continued two steps respectively in numerical calculation.

It is noted from the numerical analysis that the calculated results using Mach number checkout method and pressure checkout method were same.

As compared with the specified area method and the specified pressure method, the proposed method has higher accuracy of calculation and requires less computational time.

\section{Numerical Calculation}

\subsection{Computational Conditions}

In the present numerical analysis calculation, the basic nozzle geometry is specified as $\theta_{1}=10^{\circ}, \theta_{2}=40^{\circ}$, $r_{0}=0.006 \mathrm{~m}$ and $R=0.4 \mathrm{~m}$. Generally, $\lambda$ is taken as 0.8 . A gas-particle mixture flow is composed of hydrogen and metallic. The physical constants and the initial conditions considered in the numerical analysis are listed in Tables 1 and $2,{ }^{7)}$ respectively.

The initial value of $\alpha$ is give by

$$
\alpha_{0}=\frac{1}{1+\frac{\lambda}{1-\lambda} \frac{\rho_{\mathrm{g} 0}}{\rho_{\mathrm{p}}} \frac{U_{\mathrm{g} 0}}{U_{\mathrm{p} 0}}}
$$

The expressions of viscosity and conductivity of hydrogen can be given as a function of gas temperature as follows:

$$
K_{\mathrm{g}}=\frac{0.0125\left(1+0.000247 T_{\mathrm{g}}\right) T_{\mathrm{g}}^{0.5}}{1+\frac{69}{T_{\mathrm{g}}}}
$$

\subsection{Results and Discussion}

Figure 2 indicates the variation of gas velocities along $x$ axis of the nozzle with $\lambda$ as a parameter. Here, it should be noted that $\lambda=0$ corresponds to single-phase

Table 1. Physical constants of gas and particle.

\begin{tabular}{ll}
\hline \multicolumn{1}{c}{ Gas (hydrogen) } & \multicolumn{1}{c}{ Particle (metallic) } \\
\hline$\gamma=1.4$ & $\rho_{\mathrm{p}}=18700\left(\mathrm{~kg} / \mathrm{m}^{3}\right)$ \\
$C_{\mathrm{pg}}=14595[\mathrm{~J} /(\mathrm{kg} \cdot \mathrm{K})]$ & $\left.C_{\mathrm{s}}=117.23[\mathrm{~J} / \mathrm{kg} \cdot \mathrm{K})\right]$ \\
$R_{\mathrm{g}}=4124.16[\mathrm{~J} /(\mathrm{kg} \cdot \mathrm{K})]$ & \\
\hline
\end{tabular}

Table 2. Initial conditions of gas and particle.

\begin{tabular}{ll}
\hline Gas (hydrogen) & Particle (metallic) \\
\hline$T_{\mathrm{g} 0}=590 \mathrm{~K}$ & $T_{\mathrm{p} 0}=590 \mathrm{~K}$ \\
$U_{\mathrm{g} 0}=0.15(\mathrm{~km} / \mathrm{s})$ & $U_{\mathrm{p} 0}=0.1(\mathrm{~km} / \mathrm{s})$ \\
$\rho_{\mathrm{g} 0}=9.04\left(\mathrm{~kg} / \mathrm{m}^{3}\right)$ & $d_{\mathrm{p}}=10 \mu \mathrm{m}$ \\
$M_{0}=0.081$ & \\
$p_{0}=22 \times 10^{6}\left(\mathrm{~N} / \mathrm{m}^{2}\right)$ & \\
\hline
\end{tabular}


flow (for gas flow only), and the position of the throat is changed with $\lambda$. The length of convergent parts tend to decrease along $x$ axis with the increase of $\lambda$ (at $x_{*}=$ $27.0 \mathrm{~mm}$ for the case of $\lambda=0$, at $x_{*}=26.6 \mathrm{~mm}$ for $\lambda=0.2$, at $x_{*}=26.0 \mathrm{~mm}$ for $\lambda=0.5$ and at $x_{*}=24.1 \mathrm{~mm}$ for $\lambda=0.8$ ). It can be seen from this figure that the gas velocity does not change significantly in the convergent part, but increase with decreasing $\lambda$ in the divergent part of the nozzle.

Figures 3(a) and 3(b) indicate the variation of gas and

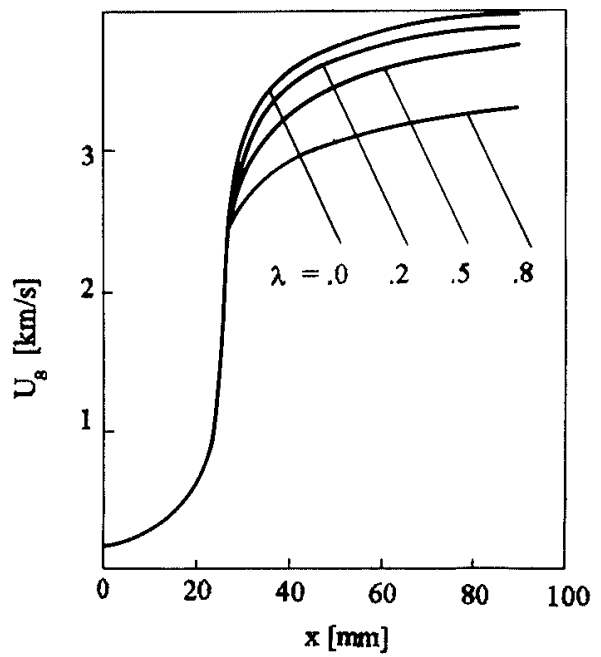

Fig. 2. Variation of gas velocities along $x$ axis with $\lambda$ as a parameter. particle velocities, respectively, along $x$ axis with different cone angles of divergent part $\left(\theta_{2}=10^{\circ}, 20^{\circ}, 30^{\circ}, 40^{\circ}\right)$. In present case, the critical angle $\theta_{c}$ is $22^{\circ}$, and the diameter of the throat of nozzle is the same for different $\theta_{2}$. When $\theta_{2} \geq \theta_{c}$, the gas velocity is subsonic in the convergent part, but supersonic in the divergent part, whereas particle velocity is increased along $x$ axis. It is noted that, when the cone angle of divergent part is equal to the critical angle, both the terminal gas and particle velocities at the exit of nozzle are maximum. When $\theta_{2}<\theta_{c}$, the gas velocity is subsonic in both convergent part and divergent part of nozzle; whereas the particle velocity increases in the convergent part and increases at first then decreases in the divergent part.

Figures 4(a) and 4(b) indicate the variation of the gas velocity and particle velocity, respectively, along $x$ axis with different radius of curvature $R$. It can be seen from these figures that gas velocity and particle velocity increase along $x$ axis. The length of convergent parts are decreased with increasing $R$. The numerical analysis shows that, when $R>5 \mathrm{~m}$, the length of convergent parts are not changed with $R$. It is noted that $R=\infty$ or $R>5 \mathrm{~m}$ is corresponding to the cone convergent part of nozzle. In this case, both the gas and particle velocities are maximum along $x$ axis. The numerical analysis also shows that the critical angles are increased with $R$ $\left(\theta_{\mathrm{c}}=18^{\circ}\right.$ for the case of $R=0.25 \mathrm{~m}, \theta_{\mathrm{c}}=22^{\circ}$ for $R=0.4 \mathrm{~m}$, $\theta_{\mathrm{c}}=23^{\circ}$ for $R=0.55 \mathrm{~m}, \theta_{\mathrm{c}}=25^{\circ}$ for $R=\infty$ ).

Figure 5 shows the variation of gas velocity along the
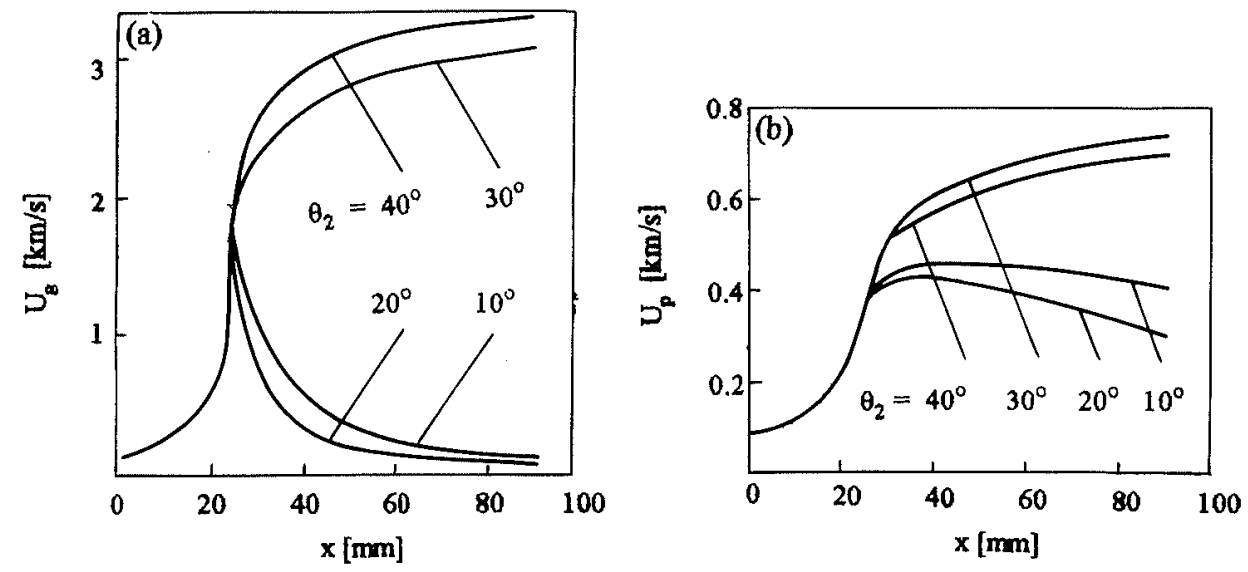

Fig. 3. Variation of gas velocities (a) and particle velocities (b) along $x$ axis with $\theta_{2}$ as a parameter.
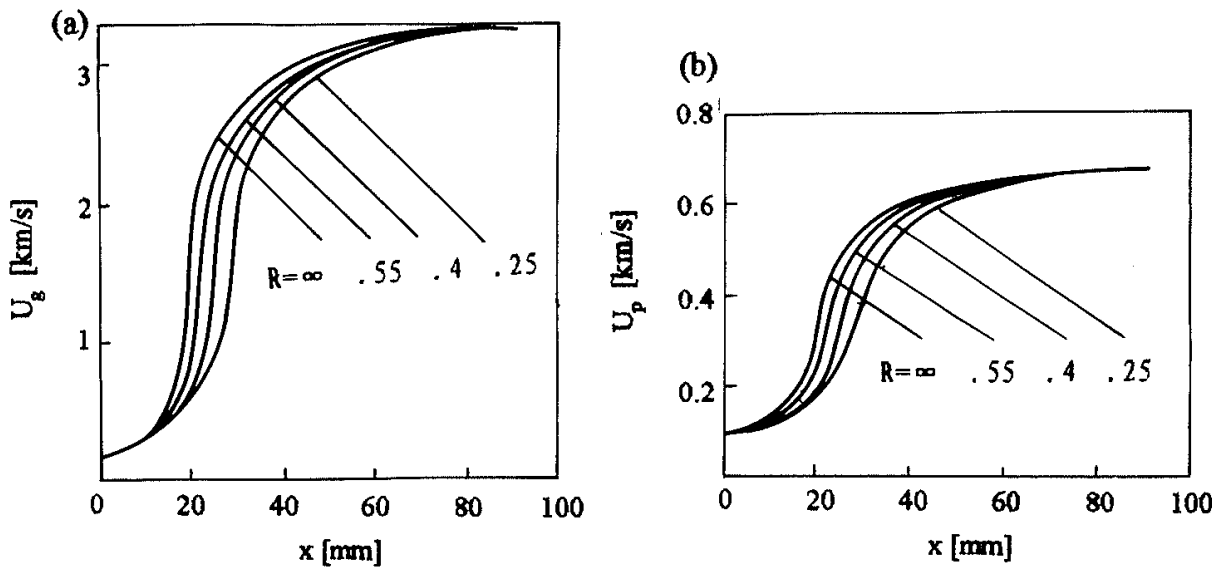

Fig. 4. Variation of gas velocities (a) and particle velocities (b) along $x$ axis with $R$ as a parameter. 


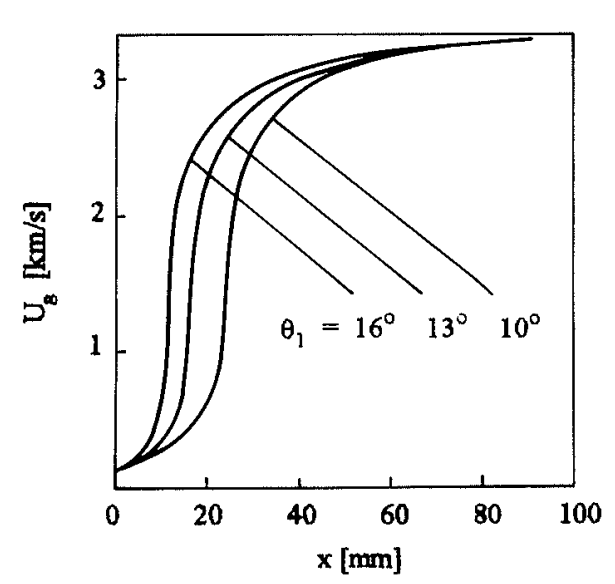

Fig. 5. Variation of gas velocities along $x$ axis with $\theta_{1}$ as a parameter.

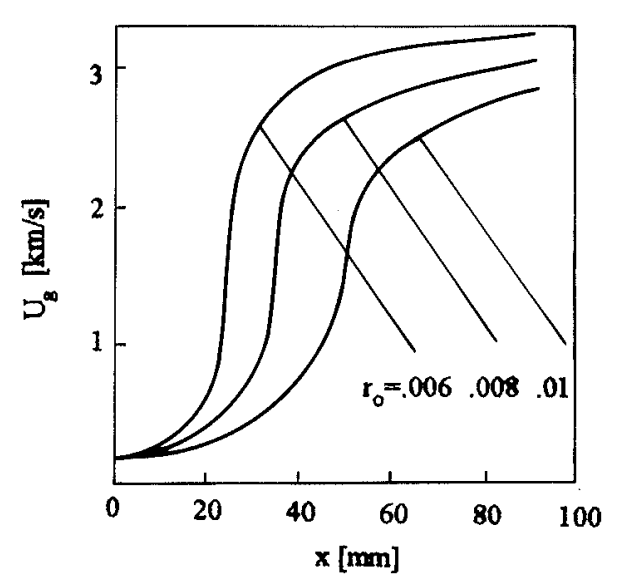

Fig. 6. Variation of gas velocities along $x$ axis with $r_{0}$ as a parameter.

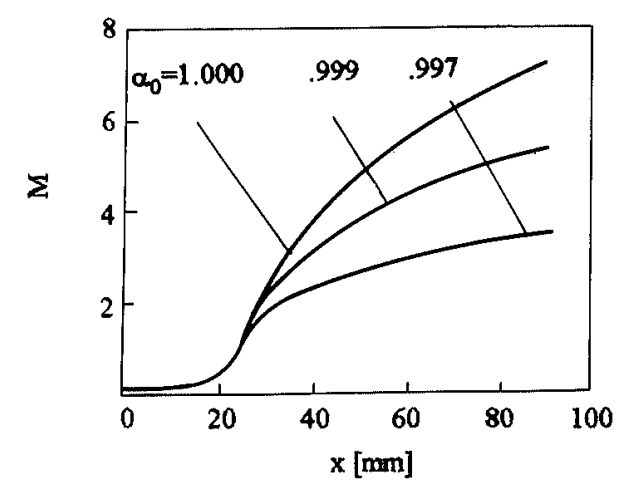

Fig. 7. Variation of Mach number along $x$ axis with $\alpha$ as a parameter.

nozzle axis with $\theta_{1}$ as a parameter. It can be seen from the figure that gas velocity increases with $\theta_{1}$, and the length of convergent parts are decreased with increasing $\theta_{1}$. The numerical analysis shows that the critical angles increase with $\theta_{1}\left(\theta_{c}=22^{\circ}\right.$ for the case of $\theta_{1}=10^{\circ}, \theta_{c}=26^{\circ}$ for $\theta_{1}=13^{\circ}, \theta_{\mathrm{c}}=29^{\circ}$ for $\theta_{1}=16^{\circ}$ ).

Figure 6 shows the variation of gas velocity along the nozzle axis with $r_{0}$ as a parameter. It can be seen from the figure that gas velocity and the length of convergent part increase with decreasing $r_{0}$. The numerical analysis shows that the critical angles increase at first, then decrease with increasing $r_{0}\left(\theta_{\mathrm{c}}=22^{\circ}\right.$ for the case of $r_{0}=0.006 \mathrm{~m}, \theta_{\mathrm{c}}=24^{\circ}$ for $r_{0}=0.008 \mathrm{~m}, \theta_{\mathrm{c}}=23^{\circ}$ for $r_{0}=0.01 \mathrm{~m}$ ).

Figure 7 indicates the variation of gas velocity along the nozzle axis with $\alpha$ as a parameter. It can be seen from the figure that the gas velocity is independent of $\alpha$ in the convergent part. Therefore, in the calculation of supersonic gas-particle flow, it is necessary to consider the influence of particle volume fraction on two phase flow when $\lambda$ is large.

According to numerical analysis, the effects of correction factor $\psi$ and correction of effective viscosity $\mu_{\mathrm{e}}$ are not significant on the flow properties in De Laval nozzle. Therefore, the effect of particle clouds on the drag between particles and gas can be considered negligible.

\section{Conclusions}

We have derived the system of equations governing a gas-particle mixture in De Laval nozzle with curved convergent part. The numerical analysis shows that the critical angles depend on the variation of $\lambda, R, \theta_{1}$, and $r_{0}$. When the angle at the divergent part is larger than the critical angle, the supersonic flow can be obtained in the divergent part of De Laval nozzle. When the angle at the divergent part is equal to the critical angle, the maximum particle velocity can be obtained.

We hope that our theoretical model can be applied to the establishment of the conditions of optimum nozzle of performance.

\section{Nomenclature}

$A$ : sectional area of nozzle

$C_{\mathrm{d}}:$ drag coefficient

$C_{\mathrm{pg}}:$ gas specific heat

$C_{\mathrm{s}}$ : specific heat of particle

$d_{\mathrm{p}}:$ particle diameter

$D$ : diameter of nozzle

$K$ : ratio of particle velocity to gas velocity

$K_{\mathrm{g}}:$ thermal conductivity of gas

$M$ : gas-phase Mach number

$N u$ : particle Nusselt number

$p$ : pressure

Pr: gas-phase Prandtl number

$r$ : radius of nozzle

$R$ : curvature radius

$R e$ : particle Reynolds number

$R_{\mathrm{g}}$ : gas constant

$T$ : temperature

$U$ : velocity

$W:$ total mass flow rate

$x$ : coordinate along nozzle axis

$\alpha$ : volume fraction of gas

$\gamma:$ ratio of specific heat

$\lambda$ : ratio of mass flow rate of particles to the total mass flow rate

$\rho:$ density

$\mu_{\mathrm{e}}$ : effective viscosity

$\mu_{\mathrm{g}}$ : viscosity of gas

$\theta_{1}$ : initial tangent angle

$\theta_{2}$ : cone angle of diverging part of nozzle

$\theta_{c}$ : critical angle 
$\psi:$ correction factor for drag coefficient

\section{Subscripts}

0 : quantity at nozzle inlet

*: quantity at nozzle throat

e: quantity at nozzle exit

g: gas-phase

$\mathrm{p}$ : particle-phase

\section{REFERENCES}

1) J. H. Neilson and A. J. Gilchrist: J. Fluid Mech., 33 (1968), 131.

2) M. J. Zucrow and J. D. Hoffman: Gas Dynamics, II, John Wiley and Sons, New York, (1977), 53.

3) N. Hatta, H: Takuda, R. Ishii and H. Fujimoto: ISIJ Int., 29 (1989), 605 .
4) S. Ishimaru and M. Nishida: Trans. Jpn. Soc. Mech. Eng., B, 55 (1989), 2262.

5) S. Ishimaru and M. Nishida: Trans. Jpn. Soc. Mech. Eng., B, 55 (1989), 2277.

6) Y. C. Peng and A. I. Kolomentsev: J. Northeast Univ. Technol., 14 (1993), 591

7) A. I. Kolomentsev and Y. C. Peng: Eng. Chem. Metall., 15 (1994), 129.

8) Y. C. Peng, B. C. Wen and A. I. Kolomentsev: J. Northeastern Univ., 15 (1994), 45.

9) Y. C. Peng and A. I. Kolomentsev: J. Northeastern Univ., 15 (1994), 152.

10) K. F. Cen and J. R. Fan: Theory and Calculation of Engineering Gas-Particle Flow, Zhejiang University Publishing House, Zhou, (1990), 390.

11) C. K. W. Tam: J. Fluid Mech., 38 (1969), 537.

12) N. Zuber: Chem. Eng. Sci., 19 (1964), 897. 